\title{
Nanoparticles and their Current Potential Use to Detect Monocyte Subsets in Vivo
}

\author{
Mauricio Rojas-López ${ }^{1,2}$ \\ ${ }^{1}$ Grupo de Inmunología Celular e Inmunogenética, Sede de Investigación Universitaria (SIU), Universidad de \\ Antioquia (UDEA), Calle 70 No. 52-21, Medellín, Colombia \\ ${ }^{2}$ Unidad de Citometría de Flujo, Sede de Investigación Universitaria (SIU), Universidad de Antioquia (UDEA), \\ Colombia
}

\begin{abstract}
Monocytes play critical roles in different inflammatory, regulatory, and tissue remodeling processes. In several diseases, an unbalanced monocyte response can occur and trigger an immunopathological disorder. In general, the characterization of monocytes in those diseases requires very invasive procedures that cannot be repeated frequently because of the health risks for patients. Here, we review the potential use of nanodiagnosis tools to discriminate monocyte subsets to the light of recent advances in the field; nevertheless, it is essential to consider the increasing complexity of the mononuclear populations that in turn, will also require new approaches for a better understanding.
\end{abstract}

Keywords: Monocyte, Nanoparticles, inflammatory diseases.

\section{Background}

Monocyte subsets: A growing unresolved complexity

Circulating human monocytes are bone marrowderived leukocytes that can differentiate into macrophages and dendritic cells. They participate in the induction and regulation of the inflammatory processes as well as in the adaptive immune responses [1]. The monocyte population is heterogeneous, exhibits high plasticity, and includes cellular subsets with different morphological features and functions. The monocyte subpopulations are conventionally defined according to the relative surface expression of CD14 (co-receptor, along with the toll-like receptor 4/TLR4, of the bacterial lipopolysaccharide/LPS), and CD16 (FcyRIIIa). Although three different monocyte subsets were initially defined: classical (CD14++CD16-), intermediate (CD14+CD16+), and non-classical (CD14+ CD16++) [2], the advances in molecular immunology have demonstrated that this cellular lineage is more complex than previously observed as it is described later.

Classical monocytes constitute about $80-95 \%$ of the circulating monocytes and have prominent phagocytic capabilities [3]. Classical monocytes are important scavenger cells [4] that remove apoptotic bodies in a non-inflammatory fashion. They are rapidly recruited to sites of infection [5] and injury [6-8], where they exhibit considerable functional plasticity [9]. The intermediate subset comprises about $2-8 \%$ of the circulating monocytes, increase in inflammatory or infectious conditions, and produce TNF- $\alpha$, IL-1 $\beta$, IL-6, and IL-10 $[10,11]$. These cells participate in the proliferation and stimulation of T-cells, the inflammatory responses, and the angiogenesis. It is proposed that intermediate monocytes make up a transitional population bridging the classical and non-classical ones [12].

Corresponding Author: Mauricio Rojas López, Grupo de Inmunología Celular e Inmunogenética, Sede de Investigación Universitaria (SIU), Universidad de Antioquia (UDEA), Calle 70 No. 52-21, Medellín, Colombia Received date: May 16, 2019; Accepted date: May 23, 2019;

Published date: May 24, 2019.

DOI: https://doi.org/10.31546/BBAB.1002 
Non-classical monocytes correspond to about 2-11\% of circulating monocytes, are considered the major patrols of the endothelium where they search for injury, and accumulate in non-inflamed peripheral tissues such as spleen, lung, and liver when adoptively transferred [13]. They can have a proinflammatory behavior and secrete inflammatory cytokines in response to infection. These cells are also involved in antigen presentation and $\mathrm{T}$-cell stimulation $[14,15]$.

Murdoch et al. [16] and Venneri et al. [17] identified two distinct subpopulations of CD16+ (intermediate and non-classical) monocytes based on the surface expression and function of the Tie-2 marker. Also, the expression of Slan (6-sulfo LacNAc) further distinguished the intermediate and non-classical monocyte subsets [18]. Nevertheless, the whole functions of the intermediate population have not been well defined since some reports suggest that they are more related to the classical monocytes and others present conflicting evidence.

In experimental murine models of cancer, compelling evidence points towards a role of tumor-associated macrophages (TAM) in tumor lymphangiogenesis. TIE-2-expressing monocytes (TEM) are highly proangiogenic immunosuppressive cells; and, TIE-2 and the receptor for the vascular endothelial growth factor (VEGFR) signaling pathways drive the TEM immunosuppressive function. TIE-2 and VEGFR signaling pathways display variable contributions to TEM angiogenic and lymphangiogenic activities in patients with breast cancer; however, the combined therapy of TIE-2 and VEGFR kinase inhibitors abrogated these activities and overcame the interpatient variability [19]. Sorafenib, a multi-kinase inhibitor, suppresses tumor angiogenesis and has become the first-line systemic therapy for patients with advanced hepatocellular carcinoma [20]. On the other hand, slan+ monocytes seem to be around $50 \%$ of the circulating CD16+ monocytes and contribute half of the cells in the inflammatory response in different diseases including lupus nephritis [21] and granulomatous inflammation [18]. However, the balance between several monocyte subsets in different pathologies requires a very systematic observation frame to determine if these cells are as they are expected to be.

Just like monocytes, macrophages cannot be defined as a homogeneous cellular population since they are found throughout the body in different shapes and functions, conditioned by the tissue where they reside. Their numbers are constantly replenished by a pool of circulating monocytes that originate either directly from the bone marrow or a reservoir within the spleen or the same tissue, as suggested by Swirski et al. [22]. The mechanisms involved in the evolution of different diseases have been studied through biomedical imaging with ex vivo labeled-monocytes and their subsequent tracking in vivo [23]. Additionally, in vivo phagocytosis of nanoparticle imaging agents have been utilized to characterize disease stages, e.g., for differentiation of benign versus malignant neoplastic lesions [24-26] and the definition of tissue macrophage infiltration in autoimmune encephalitis and multiple sclerosis [27, 28].

\section{Monocyte subsets are differentially involved in different chronic inflammatory diseases}

It is not surprising that given their multiple and pleiotropic functions, monocytes display different alterations in number, proportion, and role in several pathologies, mainly in chronic inflammatory diseases. Some of them include childhood obesity [29], diabetes [30, 31], atherosclerosis [32-34], cardiovascular diseases [35], infection by HIV [36], and Chron's disease [37]. Our Group has reported phenotypic and functional alterations in monocyte subsets in tuberculosis and functional differences that seem related to their stage of differentiation $[38,39]$. In the case of systemic lupus erythematosus (SLE), non-classical monocytes are found in the renal lesions and their role in the disease is evident [40]. Our in vitro studies showed altered proportions of circulating monocytes in patients with SLE; besides, those cells exhibited functional anomalies that suggested the loss of their regulatory functions in the proliferation of T-cells [41]. In SLE, monocytes are involved in nephritis mediated by immune complexes containing antiDNA and anti-C1q antibodies; immune complexes-mediated damage is considered one of the leading causes of mortality in patients with this disease [42].

Altered functions of monocytes are also described in rheumatoid arthritis (RA) [43]. The intermediate monocytes are increased in patients with RA, whereas the non-classical monocytes are not [10]; therefore, it is suggested that intermediate monocytes may migrate from peripheral blood into the synovia and differentiate into tissue M1 or M2 macrophages [44] to modulate the local inflammatory response. 
Although macrophages favor the host immune response, there is growing evidence that these cells can also be harmful due to either changes in their intrinsic function or the perpetuation of the inflammatory conditions. For instance, macrophages that infiltrate the tumor stroma, or Tumor-Associated Macrophages (TAMs), can operate as regulatory components that decrease the effector activities of the immune system, promote angiogenesis, and provide a supporting stroma for the carcinoma cells. The tumor may exert its effects through soluble factors that amplify macrophage progenitors away from the tumor stroma [45, 46], generate molecular gradients that attract circulating monocytes [47], produce immunoregulatory cytokines, and modulate the function of TAMs in the local tumor microenvironment [48].

Other example is the study performed by Seidler et al., in which they identify alterations of monocyte subpopulations in healthy human volunteers relative to the age. Several changes in the innate immune systems, including neutrophils, monocytes, macrophages, natural killer and natural killer $\mathrm{T}$ (NKT) cells and dendritic cells have been reported to contribute to the "immunosenescence" observed at old age $[49,50]$. This study demonstrates a substantial shift from 'classical' CD14++CD16- to 'non-classical' CD14+CD16+ monocytes with increasing age in healthy adults A similar observation had been made in the year 1999 in a small study that reported a significant expansion of CD14+CD16+ monocytes in the elderly, although both studies are not directly comparably, because the prior study compared very old (mean age 88 years) with young (mean age 30 years) subjects [51] To note, the significant increase of non-classical monocytes with a reduced surface expression of HLA-DR and CX3CR1 during aging [52] but Classical CD14++CD16monocyte counts did not vary dependent on age. In addition to this findings, Seidler et al, observed that serum concentrations of MCP-1 (CCL2), but not other chemokines, including fractalkine (CX3CL1), increased with age. These findings also highlight the broad and diverse role of monocytes under normal physiological conditions. When monocytes were differentiated into monocyte-derived macrophages, in response to LPS, accumulated high amounts of TNF $\alpha$, IL6, IL1 $\beta$, IL10 and also the chemokines MCP-1, MIP1 $\alpha$ and MIP1 $\beta$, while MIG was not significantly induced by LPS. Again, cells derived from old volun- -teer preserved functional capacity to produce these cytokines/chemokines upon LPS stimulation.

However, from the side of pathology, when it is more critical to understand what monocytes are doing, their possible functional alterations and where they are taking place, studies based on peripheral blood cells show only a part that partially reflects the alteration without realizing what may be happening in localized sites. At the early stages of many diseases, when the type of tissue damage is not evident, it is difficult to make a prognosis, and any selective intervention on macrophages is not feasible. In many cases, biopsies or bronchoalveolar lavages are required to access the compromised organ. These procedures are invasive and cannot be repeated because of the health risks for patients [53]; besides, in some cases, the injured tissue is difficult to access to.

\section{Why study phagocytes using nano approaches?}

During the last decade, several nanomaterials have been designed in different forms such as nanotubes, nanorods, and dendrimers. At the same time, the different roles of mononuclear phagocytes (MPs): monocytes, macrophages and dendritic cells (DCs) in diseases and chronic inflammatory processes have become clearer. Additionally, monocyte subsets are thought to differentially participate in the onset, evolution, and outcome of many diseases. Mononuclear phagocytes survey and protect the tissue microenvironment and secrete inflammatory, growth and regulatory factors.

Monocytes and macrophages are widely considered as interesting targets for both diagnosis and treatment of many pathologies. Monocytes are broadly distributed and have specialized functions in different tissues, exhibit positive and negative modulatory effects on the inflammatory responses, and can differentiate into macrophages and DCs. Monocytes can infiltrate solid tumors and promote the angiogenesis, remove dying cells, regulate the endothelial function, contribute to the buildup of the atheromatous plaque, participate as reservoirs for many infectious agents, and be the primary effector cells in the response against that type of microorganisms. 
The broad tissue distribution, the multiple roles, and the pleiotropic effects of MPs make relevant the question about their altered distribution and function during diseases and tissue remodeling. There is a need for a noninvasive method for assessing disease activity at the cellular level is necessary. To this purpose, nanomedicine offers new possibilities; most of them are under development and could be used to track MPs or even to address "bullet drugs" against them.

\section{Tracking of monocytes through imaging with nano tools}

Positron Emission Tomography (PET), fluorescent nanomaterials, X-ray computed tomography, and Magnetic Resonance Imaging (MRI) are some of the most common approaches used in clinical imaging. However, the detection limits of those techniques to identify a monocyte subset in a tissue involved in an inflammatory process of any origin are not clear.

PET is an imaging technique that detects pairs of gamma rays emitted by a positron-emitting radiopharmaceutical media. The most widely used media is Fluorine-18 $(18 \mathrm{~F})$, which is intravenously injected in a biologically active molecule (the radioactive tracer) and registered by external detectors that are positioned at different orientations. The isotope distributes within different tissues according to the nanocarrier and emits a positron. In turn, this positron interacts with a free electron and induces a reaction of extinction, resulting in the emission of two photons at almost 180-degree to each other that are detected by crystals often made of bismuth germanate. Electronically coupled opposing detectors identify the pair of $\gamma$ photons simultaneously by using coincidence detection circuits that measure extinction events within 10-20 ns. Nanoparticle-based PET agents frequently include modified dextrans [54-56], graft-copolymers [57, 58], and other scaffolds [59-61]. These nanomaterials are used to track inflammation based on the fact that tissue macrophages and infiltrating monocytes can phagocytose them. Those phagocytes comprise most of the cells inhabiting the tissues, help to initiate the inflammatory response by the coordinated release of mediators, and have the potential to restrain the inflammation. Additional monocytes can be recruited subsequently from the blood to the tissues where they can differentiate into inflammatory macrophages. All these cells orchestrate the inflammatory process and are likely a pivotal point in defining the resolution of the process versus its progression towards permanent damage. The interaction of macrophages with fibroblastscan lead to the production of extracellular matrix proteins that induce tissue repairing.

The 11C-labeled R-isomer of the PK11195 (isoquinoline carboxamide) which binds selectively to the Peripheral Benzodiazepine Receptor (PBR), highly abundant in macrophages [62], has been used in animal models to monitor the accumulation and clearance of lung-instilled particles. Some patients with chronic obstructive pulmonary disease or asthma show a higher than normal uptake of 11CR-PK11195 [63], similarly to cigarette smokers with asthma or sclerodermaassociated fibrosing alveolitis [64, 65]. Branley et al. [64] demonstrated the possibility to study the pulmonary inflammation in fibrosing alveolitis due to systemic sclerosis through a noninvasive procedure, by PET. Particularly, phagocytes could be tracked in the lung thanks to the expression of PBR, a marker of macrophage trafficking to the lung parenchyma. The authors found a downward trend in the uptake of $11 \mathrm{C}$ [R]-PK11195 in fibrosing alveolitis due to systemic sclerosis compared to normal lung [64]. Jarrett et al. developed a dual-mode PET/MRI active probe to target vascular inflammation. They incorporated an aliphatic amine polystyrene bead and a superparamagnetic iron oxide nanoparticle targeted to macrophages that were both coupled to positron-emitting $64 \mathrm{Cu}$ isotopes [66]; later, they demonstrated [67] that it was possible to detect the presence of macrophages in the atheromatous plaques in a murine model. Macromolecular and nanoparticle contrast agents used to target macrophages were developed and tested in three different mouse and rat models of atherosclerosis to study the vascular plaques. For multimodal detection, the probes were designed to contain gadolinium (T1 MRI) or iron oxide (T2 MRI), and 64Cu (PET). PET imaging was used to identify areas of macrophage accumulation; these areas were further tested by MRI to visualize the macrophage distribution at high resolution. In both, PET and MRI, the probes enhanced contrast at sites of vascular inflammation, but not in the healthy vessel walls. MRI was able to identify discrete sites of inflammation that were blurred together at the low resolution of PET; histological studies confirmed the presence of macrophages in the 
lesions. The multimodal imaging approach allowed a high-sensitivity and high-resolution mapping of the biomarker distribution and can become a clinical method to predict the probability of the rupture of the plaque [66, 67].

To validate that the results were not animal modelspecific, nor imaging method-dependent, PerezMedina et al. also examined rat models of vascular inflammation using the multimodal macromolecular probes (positive MRI contrast). They used the crush injury [68] and the copper-induced inflammatory reactions of rat carotid arteries [69] to ensure that the imaging approaches could label the inflamed plaques irrespective of the model. A high resolution-MR image that was zoomed in on the volume enhanced in the PET image confirmed the overlapping of the PET signal with a broad area within and around the injured tissue in the carotid artery. No PET signal associated with the uninjured contralateral side; similarly, control uninjured animals did not show any accumulation of PET signals in the vessel walls [70]. On the basis of the micro-PET imaging, Willmann et al. reported that VEGFR2-targeted microbubbles were rapidly cleared from the blood circulation (50\% blood clearance after approximately 3.5 minutes); besides, the in vivo distribution of intravenously injected N-succinimidyl-4-[18F] fluorobenzoatelabeled microbubbles showed that lung, gut, brain, and muscle were the targeted tissues. Additionally, the uptake of the targeted microbubbles was significantly higher in mouse angiosarcoma cells than in the adjacent skeletal muscle cells. Immunofluorescence staining demonstrated the accumulation of the targeted microbubbles within the hepatic Kupffer cells and the splenic macrophages [71].

The results of Willmann et al. suggested that dynamic in vivo micro-PET allowed a noninvasive study of the whole body and the tissue distribution of targeted microbubbles in mice. Targeted microbubbles were quickly removed from the blood circulation and rapidly accumulated in the liver and spleen through the phagocytosis by MPs. Contrast-enhanced ultrasonography with microbubbles targeted to molecular markers is increasingly being used in preclinical research and is likely to be translated into the clinical practice soon. The findings of Willmann et al. gave insights about the whole-body distribution of targeted microbubbles in living mice and have direct practical implications for planning and performing of future molecular imaging approaches with targeted microbubbles for contrast enhanced ultrasonography [71].

Although the methods, previously described, allow to determine the presence of tissue infiltrating phagocytes in a sensitive and specific manner, they do not identify the differential uptake of the agent by any particular type of monocyte subsets.

Hulsmans et al. [72] did not use nanoparticles directly but the PET based-strategy; notwithstanding, they correlated 18F-fluoro-2deoxyglucose (18F-FDG) uptake in hematopoietic organs with echocardiographic parameters of diastolic function in a retrospective analysis. The increased 18F-FDG positron emission signal reflected higher metabolic rates, which in marrow and spleen indicated accelerated hematopoietic activity [73]. Among patients with normal diastolic function (see Materials and Methods for patient selection criteria), they found a significant correlation of the splenic PET signal with the echocardiographic measures of filling pressures (i.e., E/e' and the estimated right ventricular systolic pressure) and observed similar trends for the marrow PET signal. These retrospective clinical data were preliminary because of the small sample size, the unspecific mechanisms behind 18F-FDG uptake, and the load dependence of echocardiographic measures acquired at different time points. Nevertheless, the parallels between the histology, flow cytometry, and imaging findings in patients and those in mice are intriguing because they indicate that the diastolic dysfunction is related with increased myelopoiesis and systemic myeloid cell oversupply.

Hulsmans et al. [72] reported that cardiac macrophages expanded and had an etiological role in diastolic dysfunction. Higher myocardial macrophage densities arose from monocyte recruitment and activation of hematopoiesis in the bone marrow and spleen. In mice with a macrophage-specific deletion of IL-10, a cytokine that increases during diastolic dysfunction, the rapid cardiac filling during diastole improves. IL-10 contributes to a shift of the macrophage phenotype towards a profibrotic one, which activates fibroblasts. Fibroblasts become more numerous and deposit collagen, leading to impaired myocardial relaxation. These data define macrophage-derived IL-10 as an autocrine profibrotic agent that promotes diast- 
-olic dysfunction. Therefore, they studied myeloid cells in two conditions that cause diastolic dysfunction in mice and compared some key findings with those in patients with heart failure with preserved ejected fraction (HFpEF). Although the clinical observations were preliminary and should be interpreted with caution, the data showed interesting similarities and some differences between the murine cohorts and humans. Cardiac macrophages expanded in both aged and SAU NA-exposed mice, and in myocardial biopsies from HFpEF patients. In both murine conditions and in patients with HFpEF, circulating monocytes, the progenitors of macrophages recruited to the injured heart were present at higher numbers. In the three scenarios, the activity in the hematopoietic organs increased [72].

Fluorescent nanoparticles that have the affinity for the MPs are used for microscopy, flow cytometry, and intravital imaging techniques such as endoscopy or optical tomography [74-76]. Indeed, fluorescent nanoparticles have yielded most of the current insights into the way macrophages and other cells process nanomaterials. A few of these preparations of nanoparticles have been well characterized and are commercially available, but the number of experimental agents abounds. One idea to keep in mind is that fluorescent nanomaterials for cell tracking usually require coat modifications of the nanoparticles that can alter their nature, and in turn, could change their interaction with MPs.

The other imaging strategy is the computed tomography (CT). This approach uses digital geometry processing for generating a $3 \mathrm{D}$ image of the internals of an object from an extensive series of twodimensional X-ray images taken around a single axis of rotation. The approach directs X-rays to an object from multiple orientations and measures the decrease in intensity along a series of linear paths.

This decrease is characterized by Beer's Law, which describes intensity reduction as a function of the Xray energy, the path length, and the material linear attenuation coefficient.

Specialized algorithms are used to reconstruct the distribution of the X-ray attenuation based on the body used to make the image. The resolution of the $\mathrm{CT}$ is typically in the submillimeter and micrometer ranges for clinical and preclinical systems respectively. In contrast to the other imaging techniques, the X-rays require relatively elevated concentrations of absorbent nanomaterials to delineate macrophages.
As a result, only a few nanoagents can be used for cell tracking at this level of resolution using CT. One of the contrast materials most widely used is ethyl-3,5-bis(acetylamino)-2,4,6-triiodobenzoate (N1177), an emulsified suspension that is composed of crystalline iodinated particles dispersed with a surfactant; N11777 particles are selectively phagocytosed by macrophages [7779]. Two biocompatible surfactants, a polyoxyethylene-polyoxypropylene block copolymer (poloxamer 338) and polyethylene glycol (PEG) are used to stabilize the particles and prevent their aggregation. Some other agents have been explored, but their use has been minimal. High atomic number materials that absorb X-rays more effectively are theoretically advantageous, but most of them are often toxic, limiting the derivation of new emulsifications for in vivo procedures. Another material used to track macrophages is PEGylated gold nanoparticles [80-83]. Dendrimer-entrapped gold nanoparticles (Au-DENPs) with polyethylene glycol (PEG) and fluorescein isothiocyanate (FI) coatings were synthesized, characterized, and studied in murine macrophages. The CCK-8 cell viability assay showed that PEGylated AuDENPs were non-cytotoxic up to $300 \mu \mathrm{M} \mathrm{Au}$. The morphology and localization of [(Au0)300G5.NHAc-FI-mPEG] DENPs were examined through silver staining, fluorescence microscopy, and Transmission Electron Microscopy (TEM). The authors found that macrophage recruitment to atherosclerotic lesions could be visualized in a noninvasive and dynamical way in live animals using micro-computed tomography or "microCT" 3D X-ray imaging [81].

Most of the strategies proposed to get the maximal resolution are based on the nuclear magnetic resonance (NMR) imaging that might allow distinguishing the tissue structures partly. This procedure is based on the signal obtained from the hydrogen nuclei contained in hydrogenrich compounds present in the cells, including water and lipids. Hydrogen possesses a property known as "spin," which depends on the number of protons. The "spin" induces a magnetic moment, generating a local magnetic field; the time to return to the initial position is designed as a relaxation time $(\mathrm{T} 1, \mathrm{~T} 2$, and $\mathrm{T} 2 *)$. MR contrast agents selectively alter the hydrogen relaxation times of tissues containing them. 
Considering that phagocytic cells must be responsible for the uptake of this material, it is necessary to define many aspects that are inherent to the image resolution: the uptake will depend on the function, number, stage, and type of activation of the phagocytes, but mainly it will depend on the monocyte subset.

In 2017, Park et al. [84] published a very detailed review about the physical background, sensitivity, and specificity of several clinically relevant imaging modalities, and provide an overview of the materials currently used for in vivo nanodiagnostics. However, the specific point regarding macrophages and subpopulations of monocytes requires a specific development for this type of cells.

Some considerations to keep in mind are the physical properties of the particle as size and charge because micrometer-sized particles are filtered in the capillary vessels. Besides, it is necessary to consider the particles hydrodynamic form during the transport through the small capillaries [85, 86]; colloidal carriers larger than $100 \mathrm{~nm}$, such as liposomes and polymeric nanospheres are endocytosed by Kupffer cells in the liver. These carriers have applications in vascular drug delivery and release, and in the sitespecific targeting (passive and active); liposomes can also activate the complement system (reviewed in [86]), whereas the splenic fenestrations filter out particles larger than $200 \mathrm{~nm}[86,87]$. The effect of surface charge in the circulation time was evaluated using magnetite-dextran particles coated with polyoxyethylene-polyoxypropylene copolymers (Synperonic: MDP particles neutral and larger in size (65.9-76.4 $\mathrm{nm}$ ) because charged particles (mainly the positive ones) tend to be cleared by the mononuclear phagocyte system more quickly and have a higher non-specific cell internalization rate than neutral particles [88]. Some experiments showed that the more the size of the particle, the more the uptake by the liver; $20 \mathrm{~min}$ post-injection of nanoparticles ( $2 \mathrm{mg}$ $\mathrm{Fe} / \mathrm{kg}$ ), the liver uptake was higher when the mean diameter increased: $22 \%$ for the smallest and $42 \%$ for the largest beads. For instance, particles formed or coated with functional groups like primary amine are readily phagocytosed compared with particles covered with carboxyl, sulfate, and hydroxyl groups [89]. Particles with a surface charge less than $15 \mathrm{mV}$ showed decreased phagocytic uptake, longer circulation time, and higher accumulation in a murine tumor model [90]. However, the surface charge of a nanoparticle can change quickly upon contact with plasma proteins that induces its adsorption and ops- -oonization [91, 92] by forming a protein envelope that favors the phagocytosis and clearance of the particles [93-95].

A common strategy for enhancing the circulation of particles is to coat them with hydrophilic and neutral polymers, such as PEG, polyethylene oxide (PEO) or polyoxyethylene (POE), according to their molecular weights [96]. PEG is a polyether compound that confers to nanoparticles resistance to protein adsorption and opsonization [97]; thus, PEG allows nanoparticles to escape from the phagocytosis by MPs and extends their circulating half-life from minutes to hours. Higher-molecular-weight PEG and a greater coating density improve the circulating half-life [96-99] of nanoparticles. For example, >100 nm dextran-coated magnetic nanoparticles functionalized by crosslinking with PEG of 5 and $20 \mathrm{kDa}$ have half-lives of 7.29 and 11.75 hours, respectively [100].

\section{Iron nanoparticles}

Except for iron oxide particles, nanomaterials have seldom been used in clinical diagnostics. Park et al. [84] considered that this is primarily due to the difficulties in attaining acceptable pharmacokinetic properties and suitable quality control, as well as to issues of toxicity, biodegradation, and clearance of the developed nanomaterials.

Representative examples of clinical, commercial and a wide range of experimental uses are listed by the National Institutes of Health Molecular Imaging and Contrast Agent Database (http://www.ncbi.nlm.nih.gov/books/NBK5330/). The idea to track or to "bullet drugs" to tissue infiltrating monocytes is not a novelty. However, tracking specific cells will require more defined details and knowledge of the cells and the nature of the bullet. Target-oriented and magnetically guided albumin nanoparticles were reviewed for their in vitro and in vivo characteristics elsewhere [101]. Magnetic particles with a low dose of doxorubicin entrapped in a carrier were characterized in a rat tail model. It is important to note that the targeting of particulate drug carriers to disease-affected tissues is considered an effective method to obtain a high local concentration of the drug as well as to reduce many unwanted side effects caused by the systemic circulation of the medicament [102]. 
The functionalization of magnetic albumin microspheres by incorporation of Protein A to bind the $\mathrm{IgGFc}$ region without chemical coupling agents has also been tested [103]; however, this strategy generate nonspecific binding and does not discard many unknown side effects of the active Protein A. The earliest reports of polycyanoacrylate and polymethylmethacrylate nanoparticles as drug targeting devices were promising [104-107]. Nanoparticles are similar to other colloidal systems that are mainly captured by MPs. However, due to their colloidal nature, they can escape the blood circulatory system via endothelial pinocytosis and endothelial fenestrations and can reach the hepatocytes [108]. Therefore, the solid core must be suitable to avoid some unwanted side effects; biocompatible superparamagnetic iron oxide (SPIO) nanoparticles with proper surface envelopes are critical for both cellular imaging and drug delivery applications.

Conventionally, SPIOs usually refers to superparamagnetic iron oxide nanoparticles with a hydrodynamic size around $100 \mathrm{~nm}$ that are taken by the reticuloendothelial system (RES), while USPIO (ultra-small SPIO) refers to particles that initially tend to escape the RES and are trapped in "deep" macrophages. Superparamagnetic iron-oxide based nanoparticles are the most extensively studied materials and are used for either nonspecific labeling or functionalization with high-affinity ligands for targeting cell surface receptors [109]. Biocompatible magnetic nanoparticles are often classified by composition, size, coating, crystallinity, and manufacturing process. Preparations (100 nm sized) are lumped as ultrasmall SPIO (USIPO) and single crystal nanoparticles as monocrystalline iron-oxide nanoparticles (MION). Iron-only nanoparticles contain both $\mathrm{Fe} 2+$ and $\mathrm{Fe} 3+$ ions in variable stoichiometric ratios [110]. The core is coated with different materials for keeping the particles in a homogeneous suspension.

The specific detection of peripheral blood-derived monocytes in human tissues requires tools of exceptional selectivity and sensitivity. The routine blood cell count allows to quantify circulating monocytes but does not inform about either the type of subset [111,112], stage of activation, or functional alteration. Additionally, the other routine available diagnostic tools for studying the specific changes of monocyte subsets in the tissue lesion are invasive approaches, precluding this way, the possibility of repeated analysis to follow up the evolution of the cellular infiltrate. Wildgruber et al. profiled the phenotypic and functional characteristics of human monocyte subsets and focused on two differential features: the shared expression of the Macrophage Colony-Stimulating Factor Receptor (MCSF-R) and the variable capacity of monocyte subsets for phagocytosis [109]. They customengineered magnetic nanoparticles and through a magnetic resonance chip technology [113] simultaneously evaluate the monocytes and monocyte-subset changes in patients with atherosclerosis. In this pathology, it is essential to monitor the macrophage functions, since those cells represent the most relevant leukocytic component of atherosclerotic plaques and contribute to lesion ruptures that result in stroke and myocardial infarction [114].

Bierry et al. [115] used MRI with SPIO gadolinium to identify the macrophages present in injured tissues of patients with infectious vertebral osteomyelitis and degenerative diskrelated inflammatory endplates. Their preliminary results about the in vivo finding of differential distribution of macrophages in these two disorders allowed them to hypothesize a possibly more accurate characterization of the vertebral endplate alterations than the classical extracellular changes detected by MR [115].

Macrophage imaging based on MR sequences has been enhanced by using USPIO particles. These particles usually have a mean diameter of 18-30 $\mathrm{nm}$ and are particularly suited for MRI of the macrophage activity. Macrophage labeling with USPIO particles has been used in inflammatory diseases as atherosclerosis, autoimmune encephalomyelitis, nephrotoxic nephritis, transplanted graft rejections, and bacterial soft-tissue infections. These particles were also used by its ability to reveal infection or joint inflammation in a model of unilateral arthritis induced by the injection of methylated bovine serum albumin into the knees of rabbits that had been previously primed with the same antigen. The MR imaging at $1.5 \mathrm{~T}$ and 24 hours after the contrast agent administration could evidence the USPIO uptake by phagocytic-active macrophages in the synovium of all the arthritic knees [116]. For clinical purposes, the ability to visualize the macrophage activity in the arthritic 
joints by MRI appears desirable in many respects. It could enable the assessment of the early stages of the disease before conventional radiography or other imaging approaches can detect structural changes. Moreover, a technique that can detect in vivo the phagocytic-active macrophages by the iron oxideassociated signal effects could be a useful tool for the surveillance of the response to therapeutical drugs that modulate the activity of macrophages in rheumatoid arthritis.

The USPIO agent (P904) (Guerbet, Aulnay-SousBois, France) was tested by Lopez-Castro et al. who used Electron Microscopy and found that these particles are degraded after being ingested by the macrophages; that is iron from the SPIO core is progressively incorporated into the iron-storing protein, ferritin, which is considered a nontoxic form of iron [117]. Nevertheless, Levy et al. reported that the superparamagnetic properties of the NPs had been modified after cell internalization, due to the magnetic interactions between NPs sequestered within intracellular organelles. The modifications in the magnetic behavior of the NPs were observed in vivo in a murine model after NP uptake by spleen and liver-resident macrophages and inflammatory macrophages in the adipose tissue, as well as in vitro in experiments with monocyte-derived macrophages. The NPs formed clusters, and their magnetic properties became dependent on the targeted organ, the dose administrated, and the time elapsed since their injection [118].

The possibility to characterize monocyte subsets using nanosensors was evaluated by Wildgruber et al. in 2009. Their first observation indicated that targeted fluorescent iron-oxide nanoparticles allowed the optical discrimination of monocyte subsets [109]. Then they engineered a putative monocyte-targeted nanoparticle by covalent attaching of anti-MCSFR antibodies to the Cross-Linked Iron Oxide (CLIO), composed by dextran-coated superparamagnetic nanoparticles [119].

The incubation of mononuclear cells for $10 \mathrm{~min}$ at room temperature with increasing doses of fluorescent-tagged CLIO-MCSFR led to similar labeling of both CD16++ and CD16+ monocyte subsets across all concentrations without detectable labeling of other cells or toxicity at doses up to 1000 $\mathrm{mg} \mathrm{Fe} / \mathrm{ml}$ as detected by Trypan blue and Annexin V stainings, in agreement with the literature [120]. Besides the fluorescence labeling, the monocyte subset was resolved with a diagnostic magnetic resonance (DMR)-chip and modeled mathematically for enumeration studies. When the phagocytic capacity was probed with CLIO, the $\Delta \mathrm{T} 2$ were higher in CD16+ monocytes compared to slight signals in cells other than monocytes [109]. Then, the authors evaluated further the clinical relevance of the CLIO labeling, through the analysis of the profile of monocyte subsets in complex physiological cell mixtures; they observed that the T2 changes of CLIOs allowed correct discrimination of the monocyte subsets [109]. The recognition of the monocytes involved in inflammatory processes promises to improve their detection in different pathologies; notably, the studies performed by Wildgruber et al. have proposed the use of CLIO for detection and treatment of patients at risk of atherothrombotic events. Their studies focused on the analysis of fluctuations of the monocyte subsets that can occur in patients with atherosclerosis. Notwithstanding, monocytes are cells of wide tissue distribution that participate in many different diseases and pathological processes, not only in the inflammatory stages but also in their resolution.

During the following two years Settles et al. [121] published evidence about the detection of monocytes by MRI. They labeled human phagocytes ex vivo, then reinjected them, and finally followed them in vivo by MRI. The reinjection of ex vivo labeled monocytes could direct the contrast agents more specifically to the injured tissue. This feature could solve the current problems of the limited sensitivity of MRI and increased the contrast-to-noise ratio due to a limited background signal. The authors exploited further these properties and showed that CD14++CD16- monocytes showed a higher uptake of both experimental and clinically approved iron oxide contrast agents and that this increased uptake led to shorter $\mathrm{T} 1$ and $\mathrm{T} 2$ relaxation times at $1.5 \mathrm{~T}$. The iron uptake of the two clinically approved iron-oxide nanoparticles, quantified by Zeeman spectrometry, was comparable to the values reported by Metz et al. [120]. The cellular uptake of MION-48 by both CD14++CD16- and CD14++CD16++ monocyte subsets was higher than the values previously reported for MION-46 by cultured peritoneal macrophages [122, 123]. This difference was attributed to the variable differentiation stage of the phagocytes as well as to different characteristics of MION-46 versus MION-48 
particles. Overall, the differences between different agents were attributed to the particle size, surface coating, surface charge, and opsonization [120, 124].

Additionally, the intracellular compartmentalization of the particles influences their relaxivity. Therefore no linear relationship can be assumed between the number of particles ingested and the relaxivity [110]. When magneto-fluorescent nanoparticles were used, the internalized iron-oxide particle (CLIO-680) colocalized with clathrin-coated vesicles. Besides, the number of intracellular nanoparticles, measured by atomic absorption spectrometry, was not directly translated into the observed relaxation times. As all of the four (Superparamagnetic iron oxide (large) or SPIO, standard SPIO (SSPIO) agents, ultrasmall SPIO (USPIO) agents and monocrystalline iron oxide nanoparticle (MION) agents) particles tested were similarly dextran coated, and the variations the in the results were mainly attributed to the particle size, opsonization, and cellular compartmentalization. Several studies have demonstrated that the endocytosis of nanoparticles is highly dependent on the particle size [125].

The results obtained by Settles et al. cannot be necessarily extrapolated to particles with other types of surface coatings. The different properties, e.g. of polyvinyl alcohol or dopamine-polyethylene-glycol coating are known to alter the interaction between the cell and the particle and the subsequent internalization of the particle [125]. However, for all particles investigated, CD14++CD16- monocytes showed higher intracellular iron concentrations compared to CD14+CD16++ ones. Also, T1 and T2 relaxation times were lower for all agents tested in CD14++CD16- monocytes compared to their CD14+CD16++ counterparts [121].

However, it is important to note that a shift of monocytes towards APCs induced by the labeling procedure itself can change the physiological course monocytes take on their route to tissue repair and homeostasis and prime the labeled cells towards a defined commitment. The increased expression of the mannose receptor CD206, which is an essential mediator of macrophage cell migration, identifies the selective differentiation of CD14+CD16++ monocytes towards the macrophage lineage just only two hours after the in-vitro labeling procedure, while CD206 does not change in CD14++CD16monocytes. The upregulation of the $\mathrm{TNF}-\alpha$ receptor CD120a in CD14++CD16- monocytes during the labeling makes this monocyte subset more susceptible to the inflammatory stimuli by $\mathrm{TNF}-\alpha$.
Although these phenotypic markers are only a few, the current results have demonstrated that even the short ex vivo labeling procedure alters the phenotype of naïve monocytes in culture and, that the up- or downregulation of cell markers differs between the two main subsets of monocytes [121].

\section{Conclusions and Perspectives}

In spite that the regulatory entities have approved different contrast agents, the uptake and usage of nanoparticles in the clinical practice are conditioned by the specific biosafety requirements that limit their use. It is important to consider that although nanomaterials can be phagocytosed or internalized by different cells, the features that allow the interaction between the phagocytes and the materials can be different depending on the inherent cell abilities for such interaction and its role in the inflammatory context.

The understanding of the inflammatory processes that involve specific monocyte subsets with different functions will require a consensus for a satisfactory identification of the cell subpopulations and their roles in the immune pathogenesis of some diseases. So, it is necessary that researches in the area also delve into the meaning, characterization, and function of the type of cellular infiltrates recently discovered.

Although the SPIO-enhanced MR imaging improves the test, the use of this type of materials will require specific training. Alternatively, in the future, this obstacle could be overcome by teleradiology strategies, that is, by referring data to a central reading staff of radiologists with expertise in interpreting this type of images.

The $\mathrm{T} 2 *$ relaxation and delta $\mathrm{T} 2 *$ often quantify the SPIO enhancement (difference of $\mathrm{T} 2 *$ relaxation before and after SPIO enhancement); the delta $\mathrm{T}_{2} *$ is assumed to correlate with the amount of SPIO. Since the findings of Settles [121] indicated that the amount of the material is critical to distinguish at least between classical and non-classical phagocytes, this measurement is strongly influenced by, among others, the static magnetic field B0 in-homogeneities; therefore, the MR technology needs to improve the $\mathrm{T}_{2} *$ quantification. Another possibility will be to exploit the T1 relaxation enhancement of 
SPIO by the T1 weighted MR sequences or the broader spread of even higher magnetic strength such as 7 Tesla; this last one will reduce the required dosage of SPIO agents for a more precise discrimination of the cells, as well as the accurate quantification of the local iron concentration. The relationship between the deltaT2* and the iron concentration is more likely to be linear when the iron concentration is low. The intracellular iron concentration seems promising for distinguishing at least the two mayor monocyte subsets and studying their distribution. In this way, it will probably be feasible to discriminate the monocyte subsets with higher selectivity.

An additional problem that can be considered is that the classification of monocytes no longer corresponds exactly to that defined by Loems Ziegler-Heitbrock $[12,18]$. The emergence of the slan+ population with proper inflammatory characteristics, in contrast to the rest of the CD16+ monocytes, also requires the development of nanotools to identify this subpopulation exclusively. In this sense, the expression of Tie-2, which does not precisely match the differential surface expression of CD16, will require a greater understanding of the biology of the slan+ cells to identify them as well as to develop tools for make them a target of the nanoparticles.

A final issue to consider is the functionalization of the nanomaterials. The fundamental problems described above need to be improved. Although several materials do not seem to compromise the viability and function of phagocytes, the most specific among them are the agonist of receptors related to the cell activation or with the antigen recognition and processing pathways. The presence of iron particles can also contribute to oxidative events in a way that has not been unveiled up to now. Coatings that reduce loads with the PEG, require additional molecules for their vectorization, and in consequence, for a cell interaction that can increase the specificity without reducing the agonist effects.

\section{Acknowledgments}

This study is part of the project entitled "Functionalization of magnetic iron oxide nanoparticles as a potential tool for tracking of tissue damage." The electromagnetic separation services were financed by the Comité para el Desarrollo de la Investigación (Committee for Development of Research/CODI by its acronym in Spanish), call 2013-2, through the Research Center of the Facultad de Ciencias Exactas y Naturales and the Programa de
Sostenibilidad (Sustainability Program) call 2012-2014 for the Cell Immunology and Immunogenetics Group at Universidad de Antioquia. We also thank Dr. Marta Mesa for their English Language Wording review, editing and correction.

\section{Conflicts of Interest: None.}

\section{References}

1. M. Guilliams, et al. Dendritic cells, monocytes and macrophages: a unified nomenclature based on ontogeny. Nat Rev Immunol. 2014; 14:571-578.

2. L. Ziegler-Heitbrock, et al. Nomenclature of monocytes and dendritic cells in blood. Blood. 2010; 116: e74-80.

3. AA. Patel, et al. The fate and lifespan of human monocyte subsets in steady state and systemic inflammation. J Exp Med. 2017; 214:1913-1923.

4. K. McCusker, J. Hoidal. Characterization of scavenger receptor activity in resident human lung macrophages. Exp Lung Res.1989; 15:651-661.

5. NV. Serbina, E.G. Pamer. Monocyte emigration from bone marrow during bacterial infection requires signals mediated by chemokine receptor CCR2. Nat Immunol. 2006; 7:311-317.

6. M. Nahrendorf, et al. High-resolution imaging of murine myocardial infarction with delayedenhancement cine micro-CT. Am J Physiol Heart Circ Physiol. 2007; 292: H3172-3178.

7. M. Nahrendorf, et al. Dual channel optical tomographic imaging of leukocyte recruitment and protease activity in the healing myocardial infarct. Circ Res. 2007; 100:1218-1225.

8. E. Zigmond, et al. Infiltrating monocytederived macrophages and resident kupffer cells display different ontogeny and functions in acute liver injury. J Immunol. 2014; 193:344-353.

9. I. Avraham-Davidi, et al. On-site education of VEGF-recruited monocytes improves their performance as angiogenic and arteriogenic accessory cells. J Exp Med. 2013; 210:26112625.

10. M. Rossol, S. Kraus, M. Pierer, C. Baerwald, U. Wagner. The CD14(bright) CD16+ monocyte subset is expanded in rheumatoid arthritis and promotes expansion of the Th17 
cell population. Arthritis Rheum. 2012; 64:671677.

11. J. Skrzeczynska-Moncznik, et al. Peripheral blood CD14high CD16+ monocytes are main producers of IL-10. Scand J Immunol. 2008; 67:152-159.

12. HW. Ziegler-Heitbrock. Heterogeneity of human blood monocytes: the CD14+ CD16+ subpopulation. Immunol Today. 1996; 17:424428.

13. F. Geissmann, S. Jung, D.R. Littman. Blood monocytes consist of two principal subsets with distinct migratory properties. Immunity. 2003; 19:71-82.

14. KL. Wong, et al. Gene expression profiling reveals the defining features of the classical, intermediate, and nonclassical human monocyte subsets. Blood. 2011;118:e16-31.

15. M. Chimen, et al. Monocyte Subsets Coregulate Inflammatory Responses by Integrated Signaling through TNF and IL-6 at the Endothelial Cell Interface. J Immunol. 2017;198:2834-2843.

16. C. Murdoch, S. Tazzyman, S. Webster, CE. Lewis. Expression of Tie-2 by human monocytes and their responses to angiopoietin-2. J Immunol. 2007; 178:7405-7411.

17. MA. Venneri, et al. Identification of proangiogenic TIE2-expressing monocytes (TEMs) in human peripheral blood and cancer. Blood. 2007; 109:5276-5285.

18. TP. Hofer, et al. slan-defined subsets of CD16positive monocytes: impact of granulomatous inflammation and M-CSF receptor mutation. Blood. 2015; 126:2601-2610.

19. S. Bron, et al. TIE-2-expressing monocytes are lymphangiogenic and associate specifically with lymphatics of human breast cancer. Oncoimmunology. 2015; 5:e1073882.

20. H. Shoji, et al. Pro-angiogenic TIE-2-expressing monocytes/TEMs as a biomarker of the effect of sorafenib in patients with advanced hepatocellular carcinoma. Int J Cancer. 2017; 141:1011-1017.

21. F. Olaru, et al. Intracapillary immune complexes recruit and activate slan-expressing CD16+ monocytes in human lupus nephritis. JCI Insight. 2018; 3(11).pii:96492.

22. FK. Swirski, et al. Identification of splenic reservoir monocytes and their deployment to inflammatory sites. Science. 2009; 325:612-616.

23. SN. Ebert, et al. Noninvasive tracking of cardiac embryonic stem cells in vivo using magnetic res- -onance imaging techniques. Stem Cells. 2007; 25:2936-2944.

24. MG. Harisinghani, et al. Noninvasive detection of clinically occult lymph-node metastases in prostate cancer. N Engl J Med. 2003; 348:2491-2499.

25. E. Seneterre, et al. Detection of hepatic metastases: ferumoxides-enhanced MR imaging versus unenhanced MR imaging and CT during arterial portography. Radiology. 1996; 200:785-792.

26. P. Soyer, Will ferumoxides-enhanced MR imaging replace CT during arterial portography in the detection of hepatic metastases? Prologue to a promising future. Radiology. 1996; 200:610-611.

27. B. Brochet, et al. Early macrophage MRI of inflammatory lesions predicts lesion severity and disease development in relapsing EAE. Neuroimage. 2006; 32:266-274.

28. V. Dousset, et al. Comparison of ultrasmall particles of iron oxide (USPIO)-enhanced T2weighted, conventional T2-weighted, and gadolinium-enhanced T1-weighted MR images in rats with experimental autoimmune encephalomyelitis. AJNR Am J Neuroradiol. 1999; 20:223-227.

29. RT. Mattos, et al. Chronic Low-Grade Inflammation in Childhood Obesity Is Associated with Decreased IL-10 Expression by Monocyte Subsets. PloS One. 2016; 11:e0168610.

30. NP. Kumar, et al. Modulation of dendritic cell and monocyte subsets in tuberculosis-diabetes co-morbidity upon standard tuberculosis treatment. Tuberculosis (Edinb). 2016; 101:191-200.

31. S. Maruo, et al. Correlation analysis of monocyte subsets and insulin resistance considering fetuin-A involvement in patients with type 2 diabetes. Clin Transl Med. 2018; $7: 9$.

32. M. Hristov, G.H. Heine. Monocyte subsets in atherosclerosis. Hamostaseologie. 2015; 35:105-112.

33. E. Idzkowska, et al. The Role of Different Monocyte Subsets in the Pathogenesis of Atherosclerosis and Acute Coronary Syndromes. Scand J Immunol. 2015; 82:163173.

34. C. von zur Muhlen, et al. Imaging monocytes with iron oxide nanoparticles targeted towards 
the monocyte integrin MAC-1 (CD11b/CD18) does not result in improved atheroscleroticplaque detection by in vivo MRI. Contrast Media Mol Imaging. 2010; 5:268-275.

35. C. Weber, et al. Role and analysis of monocyte subsets in cardiovascular disease. Joint consensus document of the European Society of Cardiology (ESC) Working Groups "Atherosclerosis \& Vascular Biology" and "Thrombosis". Thromb Haemost. 2016; 116:626637.

36. P. Chen et al. Perturbations of Monocyte Subsets and Their Association with $\mathrm{T}$ Helper Cell Differentiation in Acute and Chronic HIV-1Infected Patients. Front Immunol. 2017; 8:272.

37. S.T. Gren, O. Grip. Role of Monocytes and Intestinal Macrophages in Crohn's Disease and Ulcerative Colitis. Inflamm Bowel Dis. 2016; 22:1992-1998.

38. D. Castano, LF. Garcia, M. Rojas. Increased frequency and cell death of CD16+ monocytes with Mycobacterium tuberculosis infection. Tuberculosis (Edinb). 2011; 9:348-360.

39. ME. Ramirez-Agudelo, AC. Caro, CA. Jaramillo, M. Rojas. Fatty acid profile during the differentiation and infection with Mycobacterium tuberculosis of mononuclear phagocytes of patients with TB and healthy individuals. Cell Immunol. 2011; 270:145-155.

40. A. Barrera Garcia, et al. Infiltrating $\mathrm{CD} 16^{+}$Are Associated with a Reduction in Peripheral $\mathrm{CD} 14^{+} \mathrm{CD} 16^{++}$Monocytes and Severe Forms of Lupus Nephritis. Autoimmune Dis. 2016; 2016: 9324315.

41. C. Burbano, G. Vasquez, M. Rojas. Modulatory effects of $\mathrm{CD} 14^{+} \mathrm{CD} 16^{++}$monocytes on CD $14{ }^{++}$CD16- monocytes: a possible explanation of monocyte alterations in systemic lupus erythematosus. Arthritis Rheumatol. 2014; 66:3371-3381.

42. F. Forger, T. Matthias, M. Oppermann, H. Becker, K. Helmke. Clinical significance of antidsDNA antibody isotypes: IgG/IgM ratio of antidsDNA antibodies as a prognostic marker for lupus nephritis. Lupus. 2004; 13:36-44.

43. H. Ji, et al. Arthritis critically dependent on innate immune system players. Immunity. 2002;16:157-168.

44. J. Yang, L. Zhang, C. Yu, X.F. Yang, H. Wang. Monocyte and macrophage differentiation: circulation inflammatory monocyte as biomarker for inflammatory diseases. Biomark Res. 2014; $2: 1$.

45. V. Cortez-Retamozo, et al. Angiotensin II drives the production of tumor-promoting macrophages. Immunity. 2013; 38:296-308.

46. F. Leuschner, et al. Angiotensin-converting enzyme inhibition prevents the release of monocytes from their splenic reservoir in mice with myocardial infarction. Circ Res. 2010; 107:1364-1373.

47. BZ. Qian, et al. CCL2 recruits inflammatory monocytes to facilitate breast-tumour metastasis. Nature. 2011; 475:222-225.

48. D. Hanahan, LM. Coussens. Accessories to the crime: functions of cells recruited to the tumor microenvironment. Cancer Cell. 2012; 21:309-322.

49. JE. McElhaney, RB. Effros. Immunosenescence: what does it mean to health outcomes in older adults? Curr Opin Immunol. 2009; 21:418-424.

50. A. Panda, et al. Human innate immunosenescence: causes and consequences for immunity in old age. Trends Immunol. 2009; 30:325-333.

51. HM. Sadeghi, JF. Schnelle, JK. Thoma, P. Nishanian, J.L. Fahey. Phenotypic and functional characteristics of circulating monocytes of elderly persons. Exp Gerontol. 1999; 34:959-970.

52. S. Seidler, HW. Zimmermann, M. Bartneck, C. Trautwein, F. Tacke. Age-dependent alterations of monocyte subsets and monocyte-related chemokine pathways in healthy adults. BMC Immunol. 2010; 11:30.

53. MJ. Overman, et al. Use of research biopsies in clinical trials: are risks and benefits adequately discussed? J Clin Oncol. 2013; 13:17-22.

54. E.J. Keliher, et al. 89Zr-labeled dextran nanoparticles allow in vivo macrophage imaging. Bioconjug Chem. 2011; 22:23832389.

55. P.P. Di Mauro, V. Gomez-Vallejo, Z. Baz Maldonado, J. Llop Roig, S. Borros. Novel $18 \mathrm{~F}$ labeling strategy for polyester-based NPs for in vivo PET-CT imaging. Bioconjug Chem. 2015 ;26:582-592.

56. N.K. Devaraj, E.J. Keliher, G.M. Thurber, M. Nahrendorf, R. Weissleder. 18F labeled nanoparticles for in vivo PET-CT imaging. Bioconjug Chem. 2009; 20:397-401. 
57. K. Fukukawa, et al. Synthesis and characterization of core-shell star copolymers for in vivo PET imaging applications. Biomacromolecules. 2008; 9:1329-1339.

58. T.W. Li, $\mathrm{H}$ et al. Synthesis and in vivo pharmacokinetic evaluation of degradable shell cross-linked polymer nanoparticles with poly(carboxybetaine) versus poly(ethylene glycol) surface-grafted coatings. ACS Nano. 2012; 6:8970-8982.

59. TW. Liu, TD. MacDonald, J. Shi, BC. Wilson, G. Zheng. Intrinsically copper-64-labeled organic nanoparticles as radiotracers. Angew Chem Int Ed Engl. 2012; 51:13128-13131.

60. MS. Bradbury, M. Pauliah, P. Zanzonico, U. Wiesner, S. Patel. Intraoperative mapping of sentinel lymph node metastases using a clinically translated ultrasmall silica nanoparticle. Wiley Interdiscip Rev Nanomed Nanobiotechnol. 2016; 8:535-553.

61. M.S. Bradbury, et al. Clinically-translated silica nanoparticles as dual-modality cancer-targeted probes for image-guided surgery and interventions. Integr Biol (Camb). 2013; 5:74-86.

62. F. Zavala, M. Lenfant. Benzodiazepines and PK 11195 exert immunomodulating activities by binding on a specific receptor on macrophages. Ann N Y Acad Sci. 1987; 496:240-249.

63. HA. Jones, PS. Marino, BH. Shakur, NW. Morrell. In vivo assessment of lung inflammatory cell activity in patients with COPD and asthma. Eur Respir J. 2003; 21:567-573.

64. HM. Branley, RM. du Bois, AU. Wells, HA. Jones. PET scanning of macrophages in patients with scleroderma fibrosing alveolitis. Nucl Med Biol. 2008; 35:901-909.

65. HM. Branley, RM. du Bois, AU. Wells, HA. Jones. Peripheral-type benzodiazepine receptors in bronchoalveolar lavage cells of patients with interstitial lung disease. Nucl Med Biol. 2007; 34:553-558.

66. BR. Jarrett, B Gustafsson, DL. Kukis, AY. Louie. Synthesis of 64Cu-labeled magnetic nanoparticles for multimodal imaging. Bioconjug Chem. 2008; 19:1496-1504.

67. BR. Jarrett, C. Correa, KL. Ma, AY. Louie. In vivo mapping of vascular inflammation using multimodal imaging. PloS One. 2010; 5:e13254.

68. P. Zoldhelyi, et al. Inhibition of coronary thrombosis and local inflammation by a noncarbohydrate selectin inhibitor. Am J Physiol Heart Circ Physiol. 2000; 279:H3065-3075.
69. W. Volker, et al. Copper induced inflammatory reactions of rat carotid arteries mimic restenosis arteriosclerosis-like neointima formation. Atherosclerosis. 1997; 130:29-36.

70. C. Perez-Medina, et al. In Vivo PET Imaging of HDL in Multiple Atherosclerosis Models. JACC Cardiovasc Imaging. 2016; 9:950-961.

71. JK. Willmann, et al. Targeted microbubbles for imaging tumor angiogenesis: assessment of whole-body biodistribution with dynamic micro-PET in mice. Radiology. 2008; 249:212-219.

72. M. Hulsmans, et al. Cardiac macrophages promote diastolic dysfunction. J Exp Med. 2018; 215:423-440.

73. Y. Liu. Clinical significance of diffusely increased splenic uptake on FDG-PET. Nucl Med Commun. 2009; 30:763-769.

74. M.J. Pittet, F.K. Swirski, F. Reynolds, L. Josephson, R. Weissleder. Labeling of immune cells for in vivo imaging using magnetofluorescent nanoparticles. Nat Protoc. 2006; 1:73-79.

75. M. Nahrendorf, et al. Hybrid PET-optical imaging using targeted probes. Proc Natl Acad Sci U S A. 2010; 107:7910-7915.

76. MJ. Pittet, R. Weissleder. Intravital imaging. Cell. 2011; 147:983-991.

77. H. Zhang. Ethyl-3,5-bis(acetylamino)-2,4,6triiodobenzoate nanoparticles, Molecular Imaging and Contrast Agent Database (MICAD). Bethesda (MD). 2004-2013.

78. J.L. Van Herck, et al. Multi-slice computed tomography with N1177 identifies ruptured atherosclerotic plaques in rabbits. Basic Res Cardiol. 2010; 105:51-59.

79. F. Hyafil, et al. Quantification of inflammation within rabbit atherosclerotic plaques using the macrophage-specific CT contrast agent N1177: a comparison with 18FFDG PET/CT and histology. J Nucl Med. 2009; 50:959-965.

80. N.A. Tsyganova, et al. Penetration of pegylated gold nanoparticles through rat placental barrier. Bull Exp Biol Med. 2014; 15:383-385.

81. J. Qin, et al. Noninvasive detection of macrophages in atherosclerotic lesions by computed tomography enhanced with PEGylated gold nanoparticles. Int $\mathbf{J}$ Nanomedicine. 2014; 9:5575-5590. 
82. Z. Liu, et al. Enhancement of lipopolysaccharideinduced nitric oxide and interleukin-6 production by PEGylated gold nanoparticles in RAW264.7 cells. Nanoscale. 2012; 4:7135-7142.

83. Arnida, et al. Geometry and surface characteristics of gold nanoparticles influence their biodistribution and uptake by macrophages. Eur J Pharm Biopharm.. 2011; 77:417-423.

84. SM. Park, A. Aalipour, O. Vermesh, JH. Yu, SS. Gambhir. Towards clinically translatable in vivo nanodiagnostics. Nat Rev Mater. 2017;2(5).pii:17014.

85. E. Blanco, H. Shen, M. Ferrari. Principles of nanoparticle design for overcoming biological barriers to drug delivery. Nature biotechnology. 2015; 33:941-951.

86. SM. Moghimi, AC. Hunter, JC. Murray. Longcirculating and target-specific nanoparticles: theory to practice. Pharmacol Rev. 2001; 53:283318.

87. LT. Chen, L. Weiss, The role of the sinus wall in the passage of erythrocytes through the spleen. Blood. 1973; 41:529-537.

88. C. Chouly, D. Pouliquen, I. Lucet, J.J. Jeune, P. Jallet. Development of superparamagnetic nanoparticles for MRI: effect of particle size, charge and surface nature on biodistribution. $\mathbf{J}$ Microencapsul. 1996; 13:245-255.

89. F. Alexis, E. Pridgen, LK. Molnar, OC. Farokhzad. Factors affecting the clearance and biodistribution of polymeric nanoparticles. Mol Pharm. 2008; 5:505-515.

90. C. He, Y. Hu, L. Yin, C. Tang, C. Yin. Effects of particle size and surface charge on cellular uptake and biodistribution of polymeric nanoparticles. Biomaterials. 2010; 31:3657-3666.

91. HM. Patel, SM. Moghimi. Serum-mediated recognition of liposomes by phagocytic cells of the reticuloendothelial system - The concept of tissue specificity. Adv Drug Deliv Rev. 1998; 32:45-60.

92. A. Moore, R. Weissleder, A. Bogdanov, Jr., Uptake of dextran-coated monocrystalline iron oxides in tumor cells and macrophages. J Magn Reson Imaging. 1997; 7:1140-1145.

93. Z. Bakhtiary, et al. Targeted superparamagnetic iron oxide nanoparticles for early detection of cancer: Possibilities and challenges. Nanomedicine. 2016; 12:287-307.

94. V. Mirshafiee, R. Kim, S. Park, M. Mahmoudi, M.L. Kraft. Impact of protein pre-coating on the protein corona composition and nanoparticle cellular uptake. Biomaterials. 2016; 75:295304.

95. M. Mahmoudi, et al. Variation of protein corona composition of gold nanoparticles following plasmonic heating. Nano lett. 2014; 14:6-12.

96. C. Fang, et al. In vivo tumor targeting of tumor necrosis factor-alpha-loaded stealth nanoparticles: effect of MePEG molecular weight and particle size. Eur J Pharm Sci. 2006; 27:27-36.

97. K. Bergstrom, et al. Effects of branching and molecular weight of surface-bound poly(ethylene oxide) on protein rejection. $\mathbf{J}$ Biomater Sci Polym Ed.1994; 6:123-132.

98. JL. Perry, et al. PEGylated PRINT nanoparticles: the impact of PEG density on protein binding, macrophage association, biodistribution, and pharmacokinetics. Nano lett. 2012; 12:5304-5310.

99. EK. Larsen, et al. Accumulation of magnetic iron oxide nanoparticles coated with variably sized polyethylene glycol in murine tumors. Nanoscale. 2012; 4:2352-2361.

100. AJ. Cole, et al. Polyethylene glycol modified, cross-linked starch-coated iron oxide nanoparticles for enhanced magnetic tumor targeting. Biomaterials. 2011; 32:2183-2193.

101. AE. Senyei, KJ. Widder. Drug targeting: magnetically responsive albumin microspheres--a review of the system to date. Gynecol Oncol. 1981; 12:1-13.

102. AE. Senyei, SD. Reich, C. Gonczy, KJ. Widder. In vivo kinetics of magnetically targeted low-dose doxorubicin. J Pharm Sci. 1981; 70:389-391.

103. KJ. Widder, AE. Senyei, H. Ovadia, PY. Paterson. Specific cell binding using staphylococcal protein A magnetic microspheres. J Pharm Sci. 1981; 70:387389.

104. F. Brasseur, et al. Actinomycin D absorbed on polymethylcyanoacrylate nanoparticles: increased efficiency against an experimental tumor. Eur J Cancer. 1980; 16:1441-1445.

105. J. Kreuter, HR. Hartmann. Comparative study on the cytostatic effects and the tissue distribution of 5-fluorouracil in a free form and bound to polybutylcyanoacrylate nano 
particles in sarcoma 180-bearing mice. Oncology. 1983; 40:363-366.

106. P. Couvreur, B. Kante, L. Grislain, M. Roland, P. Speiser. Toxicity of polyalkylcyanoacrylate nanoparticles II: Doxorubicin-loaded nanoparticles. J Pharm Sci. 1982; 71:790-792.

107. B. Kante, et al. Toxicity of polyalkylcyanoacrylate nanoparticles I: Free nanoparticles. J Pharm Sci. 1982; 71:786-790.

108. J. Kreuter, U. Tauber, V. Illi. Distribution and elimination of poly(methyl-2-14C-methacrylate) nanoparticle radioactivity after injection in rats and mice. J Pharm Sci. 1979; 68:1443-1447.

109. M. Wildgruber, et al. Monocyte subset dynamics in human atherosclerosis can be profiled with magnetic nano-sensors. PloS One. 2009; 4:e5663.

110. YX. Wang, SM. Hussain, GP. Krestin. Superparamagnetic iron oxide contrast agents: physicochemical characteristics and applications in MR imaging. Eur Radiol. 2001; 11:23192331.

111. R. Dragu, et al. Predictive value of white blood cell subtypes for long-term outcome following myocardial infarction. Atherosclerosis. 2008; 196:405-412.

112. DF. Waterhouse, RA. Cahill, F. Sheehan, C. McCreery. Prediction of calculated future cardiovascular disease by monocyte count in an asymptomatic population. Vasc Health Risk Manag. 2008; 4:177-187.

113. H. Lee, E. Sun, D. Ham, R. Weissleder. ChipNMR biosensor for detection and molecular analysis of cells. Nat Med. 2008; 14:869-874.

114. K.J. Moore, I. Tabas. Macrophages in the pathogenesis of atherosclerosis. Cell. 2011; 145:341-355.

115. G. Bierry, et al. Cellular magnetic resonance imaging for the differentiation of infectious and degenerative vertebral disorders: preliminary results. J Magn Reson Imaging. 2009; 30:901906.
116. A.M. Lutz, et al. Detection of synovial macrophages in an experimental rabbit model of antigen-induced arthritis: ultrasmall superparamagnetic iron oxideenhanced MR imaging. Radiology. 2004; 233:149-157.

117. J.D. Lopez-Castro, et al. From synthetic to natural nanoparticles: monitoring the biodegradation of SPIO (P904) into ferritin by electron microscopy. Nanoscale. 2011; 3:4597-4599.

118. M. Levy, et al. Nanomagnetism reveals the intracellular clustering of iron oxide nanoparticles in the organism. Nanoscale. 2011; 3:4402-4410.

119. P. Wunderbaldinger, L. Josephson, R. Weissleder. Crosslinked iron oxides (CLIO): A new platform for the development of targeted MR contrast agents. Acad Radiol. 2002; 9:S304-S306.

120. S. Metz, et al. Capacity of human monocytes to phagocytose approved iron oxide MR contrast agents in vitro. Eur Radiol. 2004; 14:1851-1858.

121. M. Settles, et al. Different capacity of monocyte subsets to phagocytose iron-oxide nanoparticles. PloS One. 2011; 6:e25197.

122. R. Weissleder, H.C. Cheng, A. Bogdanova, A. Bogdanov, Jr. Magnetically labeled cells can be detected by MR imaging. J Magn Reson Imaging. 1997; 7:258-263.

123. T.T. Shen, A. et al. Magnetically labeled secretin retains receptor affinity to pancreas acinar cells. Bioconjugate chem. 1996; 7:311-316.

124. L. Matuszewski, et al. Cell tagging with clinically approved iron oxides: feasibility and effect of lipofection, particle size, and surface coating on labeling efficiency. Radiology. 2005; 235:155-161.

125. A. Verma, F. Stellacci. Effect of surface properties on nanoparticle-cell interactions. Small. 2010; 6:12-21. 